\title{
Ultrasound Evaluation of Pregnancy in the First Trimester
}

\section{George M Graham III}

Assistant Professor, Division of Maternal Fetal Medicine, Department of Obstetrics, Gynecology and Women's Health John A Burns School of Medicine, University of Hawaii, Honolulu, Hawaii, USA

Correspondence: George M Graham III, Department of Obstetrics, Gynecology and Women's Health, John A Burns School of Medicine, University of Hawaii, 1319 Punahou Street, Suite 540, Honolulu, Hawaii 96826, USA, Phone: (808)983-8559 Fax: (808)983-8989, e-mail: ggraham@hawaii.edu

\begin{abstract}
Improvements in ultrasound technology, including transvaginal sonography and higher frequency probes, have led to a better understanding of early pregnancy development. These advances and the increasing availability of ultrasound allow women to have an earlier and more accurate assessment of their pregnancy. First trimester sonographic signs have been identified that can be used to reassure women that their pregnancy is progressing normally or counsel them that their pregnancy will fail. In addition, first trimester ultrasound can accurately predict the type of twinning in multiple gestations, allowing for appropriate counseling and management.

Objectives

- Know the first trimester ultrasound findings of a normal intrauterine pregnancy

- Understand the ultrasound findings that diagnose an early pregnancy failure

- Know the ultrasound criteria used to diagnose a multifetal gestation

Keywords: Ultrasound, first trimester, viability, multiple gestation.
\end{abstract}

\section{INTRODUCTION}

The first trimester of pregnancy starts with the first day of the last menstrual period (LMP) and continues until the end of the twelfth week. Although, conception normally occurs two weeks after the LMP, the LMP is chosen as the start of the first trimester because it is a recognizable sign for most women. The term menstrual age and gestational age are used interchangeably, but should not be confused with embryologic age which begins with conception and is therefore 2 weeks less than menstrual and gestational age.

The first trimester can be divided into four stages: ovulation, conceptus, embryonic, and fetal. The ovulation stage occurs during the first two weeks after the LMP. This stage is followed by the conceptus stage which takes place between the third and the fifth week. The conceptus stage starts with fertilization which normally occurs within one day of ovulation, or day 15 of a 28 day menstrual cycle. It is during the conceptus stage that implantation of the blastocyst into the endometrium occurs. This may result in vaginal bleeding that can be confused with an irregular menstrual cycle. By the end of the fourth week a measurable amount of human chorionic gonadotropin (hCG) is produced, resulting in a positive pregnancy test. At this point the products of conception measure between 2 to 3 millimeters (mm), which is at the threshold of detection using transvaginal ultrasound. By the fifth week, the final week of the conceptus stage, the products of conception have formed a gestational sac that usually measures $5 \mathrm{~mm}$ in diameter. While a yolk sac may be identified at this stage, the embryo remains below the level of detection using current ultrasound technology. The embryonic period starts at the beginning of the sixth week and is the time of development. During this period the embryo first becomes visible sonographically. The organ systems start to develop and cardiac activity becomes visible by the beginning of the sixth week. The embryonic period is complete at the end of tenth week. At this time the crown rump length (CRL) of the embryo measures $30 \mathrm{~mm}$. The final two weeks of the first trimester are known as the fetal stage. This is a period of continued growth and development.

This review will focus on the use of ultrasound during these stages of development, in particular the ultrasound findings that can be used to confirm a viable intrauterine pregnancy will be outlined. In addition the role of ultrasound in the diagnosis of multifetal gestations will be reviewed. Other uses of first trimester ultrasound, such as aneuploidy risk assessment, the early anatomical survey, and threedimensional ultrasound, are not covered in this review. 
Table 1: Current indications for first trimester ultrasonography

- To confirm the presence of an intrauterine pregnancy

- To evaluate a suspected ectopic pregnancy

- To define the cause of vaginal bleeding

- To evaluate pelvic pain

- To estimate gestational age

- To diagnose or evaluate multiple gestations

- To confirm cardiac activity

- As an adjunct to chorionic villus sampling, embryo transfer, or localization and removal of an intrauterine device

- To evaluate maternal pelvic masses or uterine abnormalities

- To evaluate suspected hydatidiform mole

Adapted from American College of Obstetricians and Gynecologists. Ultrasonography in pregnancy. ACOG Practice Bulletin No. 58. Obstet Gynecol 2004;104:1449-58.

Current indications for first trimester are listed in Table $1 .{ }^{1}$ First trimester ultrasonography can be performed using either a transabdominal or transvaginal approach. The introduction of transvaginal ultrasound in the 1980s has led to major advances in first trimester ultrasound. Improved imaging is achieved with higher frequency transducers that provide better resolution but with less penetration. Transvaginal ultrasound allows the probe to be positioned closer to the uterus and therefore higher frequency probes can be used. These advantages result in improved spatial resolution and near field focusing compared with transabdominal sonography. Consequently, the findings of an intrauterine pregnancy can be seen at an earlier gestational age and a lower level of human chorionic gonadotropin (hCG) using transvaginal ultrasound. Although, the structures that confirm an intrauterine pregnancy can be equally visualized using transabdominal and transvaginal sonography after 42 days of gestation, the higher resolution transvaginal probes allow for earlier assessments of fetal viability and anatomy. ${ }^{2}$ Most of the studies involving transvaginal ultrasound used probes with a transducer frequency of 5 to 7.5 megahertz (MHz), as compared to an abdominal probe with a frequency of 3 to $7 \mathrm{MHz}$. Newer transvaginal transducers with frequencies of $10 \mathrm{MHz}$ or higher provide further improvements in spatial resolution and will likely improve the diagnostic capabilities of first trimester ultrasound.

Ultasonography in the first trimester should include a thorough evaluation of the uterus and adnexa for the presence of a gestational sac. If a gestational sac is seen, the presence of a yolk sac or embryo should be noted. The gestational age should be confirmed by the length of the embryo. If an embryo is not visualized, then the mean sac diameter should be used as a measure of gestational age. The presence or absence of cardiac activity should be reported. In multiple gestations the number of fetuses, as well as the amnionicity and chorionicity should be documented. Lastly, the uterus and adnexa should be evaluated for the presence, location, and size of any fibroids or masses, and the presence of fluid in the cul-de-sac should be noted. ${ }^{1}$

\section{NORMAL ULTRASOUND FINDINGS IN THE FIRST TRIMESTER}

\section{Gestational Sac}

The first unequivocal sign of an early intrauterine pregnancy is the gestational sac which is normally located in the mid to upper portion of the uterine cavity. The gestational sac appears as an anechoic center surrounded by an echogenic rim (Figure 1). The echogenic ring represents the developing chorionic villi and the fluid contained within the sac is chorionic fluid, so a more accurate name for this structure is the chorionic sac. ${ }^{3}$ The echogenicity of the surrounding rim should exceed that of the myometrium and should measure at least $2 \mathrm{~mm}$ thick. ${ }^{4}$ Gestational sacs are usually round early in the pregnancy, but as the sac grows it often appears more elliptical. Abnormalities of the uterine cavity, as caused by fibroids, contractions, or blood collections, can result in an irregular appearing gestational sac.

Because a gestational sac can have an elliptical or irregular shape, it is necessary to calculate the mean sac

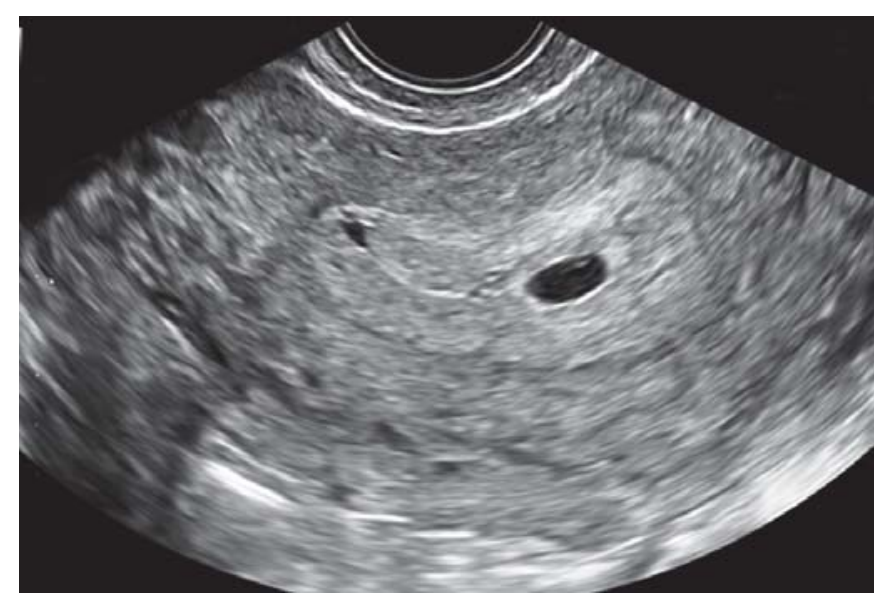

Figure 1: Normal appearing gestational sac with a double decidual sac sign 
diameter (MSD). The MSD is obtained by adding the measurements of the anechoic portion of the gestational sac in the three planes - the anteroposterior and craniocaudal diameters obtained on the sagittal view of the uterus, and the transverse diameter obtained on the transverse viewand dividing by three. It is important to measure just the anechoic portion which represents the chorionic cavity, and not to include the surrounding echogenic ring which represents the developing chorionic villi and adjacent decidual tissue. $^{4}$

A transvaginal ultrasound with a frequency of $5 \mathrm{MHz}$ or more can usually detect structures that measure at least 2 to $3 \mathrm{~mm}$, and it is at this size that the gestational sac is first appreciated. A gestational sac measuring 2 to $3 \mathrm{~mm}$ corresponds to a gestational age of 4 weeks and 1 to 3 days. The threshold for detection using transabdominal ultrasound is higher, so for this reason the gestational sac is not seen consistently until a MSD of $5 \mathrm{~mm}$, which corresponds to a gestational age of 5 weeks. ${ }^{4}$

In early pregnancy when only a small gestational saclike structure is seen, it is often necessary to distinguish a true gestational sac from a pseudogestational sac associated with an ectopic pregnancy. Before the widespread use of transvaginal ultrasound, attempts were made to identify the transabdominal ultrasound findings that confirmed an intrauterine pregnancy. The intradecidual sign was first introduced by Yeh, et al, in 1986. ${ }^{5}$ The sign is based on the concept that a normally implanted gestational sac will not displace or deform the central cavity complex. The intradecidual sign was reported to have a sensitivity of $92 \%$ and specificity of $100 \%$ for distinguishing an early intrauterine pregnancy from an ectopic. However, subsequent studies using transvaginal ultrasound by Laing et al, did not reproduce these results - the intradecidual sign resulted in a sensivity of 34 to $66 \%$ and a specificity of 55 to $73 \% .{ }^{6}$ So at this time, with the availability of transvaginal ultrasound, an intradecidual sign is not considered diagnostic of an intrauterine pregnancy. A more reliable sign for diagnosing a true gestational sac before a yolk sac or embryo is seen is the double decidual sac sign, as described by Bradley et al, in $1982^{7}$ (Figure 1). The double decidual sac sign is formed when the growing gestational sac deforms the central cavity echo complex resulting in two concentric echogenic lines that surround a portion of the gestational sac. The inner line represents the decidua capsularis and the outer line represents the decidua parietalis. A potential space exists between these two layers that often contains a small amount of endometrial fluid. The double decidual sac sign can be seen between 4 to 6 weeks using transabdominal ultrasound, and is useful for confirming an intrauterine pregnancy, before a yolk sac can be seen. However, transvaginal ultrasound can almost always identify a yolk sac at this gestational age, which limits the double decidual sac sign to transabdominal exams. ${ }^{8}$

\section{Yolk Sac}

The yolk sac is a round structure with an anechoic center and an echogenic rim, and is the first structure that can be identified within a true gestational sac (Figure 2). The yolk sac grows in size to reach its maximum diameter of 5 to 6 $\mathrm{mm}$ at 10 weeks gestation, and then gradually decreases. ${ }^{9}$ Its presence is diagnostic of a true gestational sac, with a positive predictive value of $100 \% .^{10}$

Using transvaginal ultrasound the yolk sac may first be visualized at a MSD of $5 \mathrm{~mm}$, which corresponds to a gestational age of 5 weeks, and should be seen when the MSD measures $8 \mathrm{~mm}$, which corresponds to 5.5 weeks. ${ }^{11}$ Using transabdominal ultrasound, the yolk sac should be seen when the MSD measures $20 \mathrm{~mm}$, which corresponds to a gestational age of 7 weeks. ${ }^{12}$

In early pregnancy, a small embryo at the threshold of detection can often be identified by its close approximation to the more obvious yolk sac. Similary, the yolk sac is helpful for identifying fetal cardiac activity before an embryo can be clearly identified. As the embryo develops it moves away from the yolk sac, but remains attached by the vitelline duct, also known as the omphalomesenteric duct. ${ }^{4}$

\section{Embryo}

The embryo is identifiable, when it reaches the threshold of detection, which is 1 to $2 \mathrm{~mm}$ using transvaginal sonography. At this size the embryo appears as a focal thickening adjacent to the yolk sac (Figure 3). The embryo can first be visualized

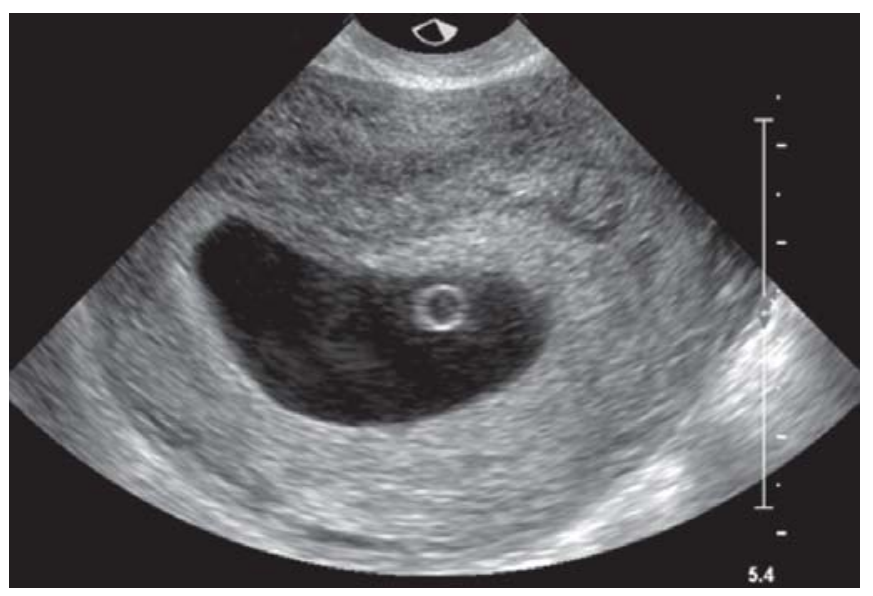

Figure 2: Normal appearing yolk sac 


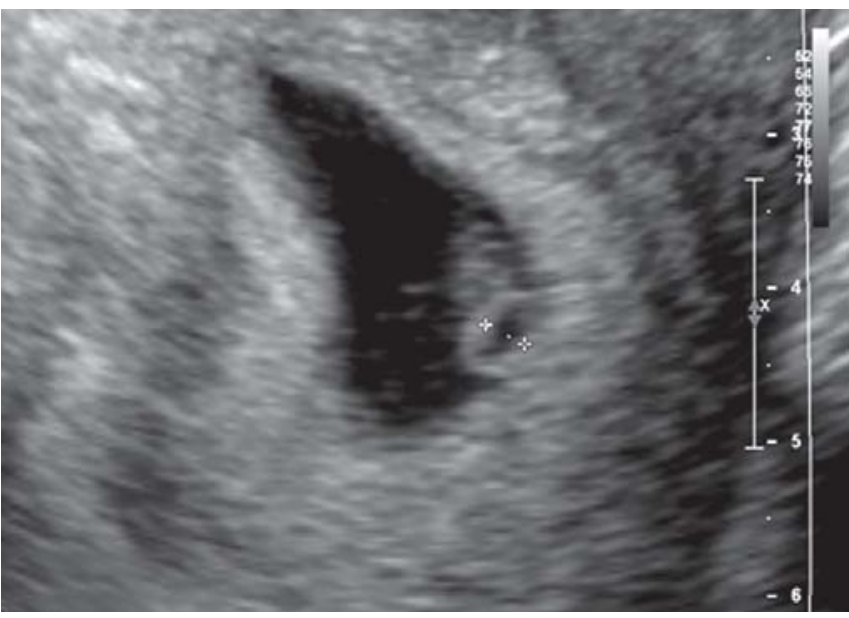

Figure 3: Early embryo adjacent to a measured yolk sac

when the MSD measures between 5 to $12 \mathrm{~mm}$. This corresponds to a GA of 5 to 6 weeks. During the 6 th week of development the embryo folds into a three-dimensional C-shaped structure and it is not possible to distinguish the crown from the rump at this early gestational age. ${ }^{4}$

\section{Cardiac Activity}

Transvaginal sonography can detect cardiac activity as early as 34 days which corresponds to an embryonic length of $1.6 \mathrm{~mm} .{ }^{13}$ Cardiac activity should be detected when the embryo measures between 4 to $5 \mathrm{~mm}$. At this time the MSD should measure between 13 to $18 \mathrm{~mm}$, which corresponds to a GA of 6 to 6.5 weeks. Using transabdominal ultrasound cardiac activity should be seen when the MSD measures $25 \mathrm{~mm}$, which corresponds to a GA of 8 weeks. The embryonic heart rate normally increases between 6 and 9 weeks gestation and then slowly decreases. Prior to 6 weeks, the heart rate is normally between 100 and 115 beats per minute (BPM). The heart rate increases and peaks at 8 weeks at 144 to 159 BPM. By 9 weeks the heart rate plateaus at 137 to $144 \mathrm{BPM}$. At this early gestational age there is almost no variation in the heart rate, so that a single measurement is acceptable by M mode. ${ }^{4}$

\section{Normal Growth}

During the embryonic period the CRL increases by approximately $1 \mathrm{~mm}$ per day and there is dramatic growth and development. ${ }^{14}$ Between 7 and 8 weeks the limb buds develop. Body and limb movement is first noted between 8 and 9 weeks. Midgut herniation into the umbilical cord begins at 9 weeks and is completed by the end of the 11 th week. ${ }^{15}$

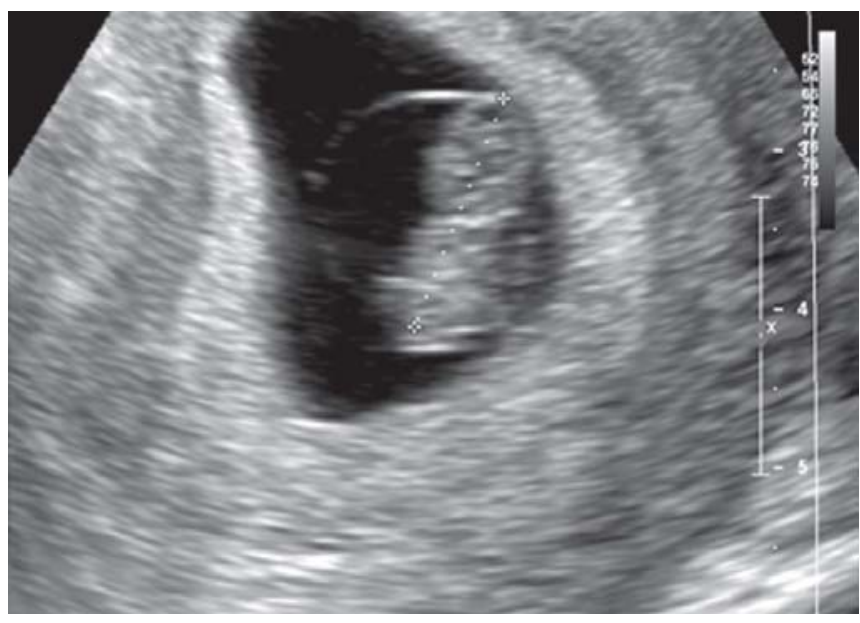

Figure 4: Amniotic membrane encircling the developing embryo

The amniotic membrane encircles the developing embryo (Figure 4). It is first visualized when the embryo is $5 \mathrm{~mm}$ in length and should be seen when the CRL measures $7 \mathrm{~mm}$ or more. Because the amniotic membrane is so thin, it is often only possible to visualize that portion that is perpendicular to the ultrasound beam. Inability to visualize the amnion, however, does not predict pregnancy failure. Initially, the CRL and the mean diameter of the amniotic cavity both increase by $1 \mathrm{~mm}$ per day and their measurements are equivalent. ${ }^{16}$ As the amniotic cavity continues to grow, it eventually fills the chorionic cavity and the two membranes become apposed. Apposition of the amnion and chorion is usually complete by 12 to 16 weeks. ${ }^{4}$

\section{DETERMINING GESTATIONAL AGE}

The estimated date of confinement is based on the assumption that pregnancy lasts 280 days from the first day of the last menstrual period. However, menstrual dating is often inaccurate because of variations in the follicular phase of the menstrual cycle and poor recall of menstrual dates by some patients. It is now accepted that the most accurate time to date a pregnancy is during the first trimester. Studies have shown that dating by CRL in the first trimester reduces the number of pregnancies considered to be greater than 41 weeks gestation and reduces post-term labor induction rates. ${ }^{17}$

Early in the first trimester there is little variability in ultrasound findings so that accurate dating is possible. When a gestational sac is seen by transvaginal ultrasound, but no yolk sac or embryo, the gestational age is 5 weeks. ${ }^{18}$ Between 5 and 11 weeks, the GA in days can be calculated by adding 
Table 2: Guidelines for dating a pregnancy during the first trimester

Stage of development

Gestational sac

(no yolk sac or embryo)

Gestational sac and yolk sac

(no embryo)

Gestational sac and yolk sac (embryo that is too small to measure with cardiac activity)

6.0

Adapted from Laing FC, Frates MC. Ultrasound evaluation during the first trimester of pregnancy. In Callen, PW (Ed): Ultrasonography in Obstetrics and Gynecology, 4th (ed), Philadelphia: WB Saunders 2000;105-45.

30 to the MSD in mm. ${ }^{4}$ Using this formula, a gestational sac with a mean diameter of $5 \mathrm{~mm}$ corresponds to a gestational age of 35 days. The identification of a yolk sac within a gestational sac, but no embryo or cardiac activity, is consistent with 5.5 weeks. Once cardiac activity is visualized but the CRL is too small to measure, the pregnancy is 6 weeks ${ }^{18}$ (Table 2). Between 6 and 12 weeks, the CRL is the most accurate method of dating a pregnancy. The CRL can estimate the EDC with an error of \pm 4.7 days. ${ }^{19}$ Strictly speaking, the length of the fetus is determined by measuring the maximal straight-line length of the fetus not the CRL. The average CRL from three satisfactory images should be averaged to predict the gestational age. In a normal pregnancy the CRL increases $1 \mathrm{~mm}$ per day, so the gestational age in days can be calculated by adding 42 to the CRL in mm. When the GA is between 43 and 67 days, this formula has a $95 \%$ confidence limit of \pm 3 days. ${ }^{20}$ By the end of the first trimester, measurements of the biparietal diameter, head circumference, abdominal circumference, and femur length are preferred for calculating gestational age.

\section{DIFFERENTIATING A NORMAL FROM AN ABNORMAL PREGNANCY IN THE FIRST TRIMESTER}

Ultrasound findings, combined with the clinical presentation and serum hCG, can be used to differentiate a normal from an abnormal pregnancy in the first trimester. There are a number of different terms that are used to describe an abnormal intrauterine pregnancy. As these different terms have little impact on clinical management, some have advocated labeling all abnormal intrauterine pregnancies in the first trimester as unsuccessful or failed pregnancies. Other diagnoses that should be considered when evaluating a potentially abnormal pregnancy are ectopic pregnancy and gestational trophoblastic disease. ${ }^{4}$
In general, ultrasound evaluation of an early intrauterine pregnancy can reveal one of three things: An empty uterus, a sac without an embryo, and a sac containing an embryo.

\section{Empty Uterus}

If ultrasound reveals an empty uterus then the endometrium should be evaluated. An abnormally thick or irregularly echogenic endometrium may represent intrauterine blood or retained products of conception, while a thin endometrium suggests a complete abortion.

A thickened regular endometrium suggests a decidualized endometrium, which is seen in any pregnancy regardless of its location. The absence of an intrauterine sac with a decidualized endometrium is consistent with a either a normal or abnormal early intrauterine pregnancy, or an ectopic pregnancy. The thickness of the endometrium does not help distinguish between these diagnoses. In these cases the quantative hCG level may be helpful.

A quantative hCG level below the discriminatory level, the lowest value for which an intrauterine gestational sac should be seen, is consistent with a normal early pregnancy, but does not rule-out an abnormal intrauterine or ectopic pregnancy. The discriminatory value for detecting an intrauterine gestational sac by transvaginal ultrasound is reported to be between 1000 to $2000 \mathrm{mIU} / \mathrm{ml}^{4}{ }^{4}$ If the suspicion for an ectopic pregnancy is low based on the patient's risk factors, symptoms, physical exam, and a thorough ultrasound exam, and the pregnancy is desired, then the patient may be managed with serial quantative hCG levels. An hCG that rises by at least $50 \%$ in 48 hours is consistent with a normal intrauterine pregnancy. In this case, a repeat ultrasound may be performed when the hCG exceeds the discriminatory value. Once the discriminatory value is exceeded, a normal intrauterine pregnancy should be detectable by ultrasound. It is important to continue to follow the patient clinically during this period for any suggestion of an ectopic pregnancy. If the pregnancy is undesired, uterine evacuation can be helpful, as the identification of chorionic tissue excludes an ectopic pregnancy.

When the quantative hCG exceeds the discriminatory level and an intrauterine sac is not seen, a normal intrauterine pregnancy is unlikely and, therefore, an abnormal intrauterine or ectopic pregnancy should be suspected. Again, uterine evacuation may be helpful to exclude an ectopic pregnancy. If chorionic tissue is not obtained, an ectopic pregnancy should be strongly suspected. 


\section{Empty Intrauterine Sac}

The identification of an empty intrauterine sac is consistent with either a normal early intrauterine pregnancy, an abnormal pregnancy, or a pseudogestational sac of an ectopic pregnancy. A pseudogestational sac is an endometrial fluid collection surrounded by a decidualized endometrium, and can be seen in 5 to $10 \%$ of ectopic pregnancies. ${ }^{8}$ Identification of the "intradecidual sign" or the "double decidual sac sign" may increase the likelihood of an intrauterine pregnancy, however a follow-up ultrasound should be obtained to confirm the diagnosis. ${ }^{5,7}$

With an abdominal probe a yolk sac should be seen when the MSD is $20 \mathrm{~mm}$ or more, and an embryo with cardiac activity should be seen when the MSD is $25 \mathrm{~mm}$ or more. ${ }^{12}$ Using a higher frequency transvaginal probe, a yolk sac should be seen when the MSD is $8 \mathrm{~mm}$ or more, and cardiac activity should be detected when the MSD exceeds $16 \mathrm{~mm} .{ }^{11}$ Some authors advocate using $18 \mathrm{~mm}$ as the discriminatory sac size for cardiac activity because of the occasional exceptions that have been seen using $16 \mathrm{~mm} .{ }^{21}$ It should be noted that these discriminatory values are suggested only as guidelines. If certain findings are not observed when expected it is always better to obtain a follow-up exam rather than misdiagnosing a normal pregnancy.

The MSD of a normal gestational sac will increase by $1.13 \mathrm{~mm} /$ day, whereas an abnormal gestational sac will increase by $0.7 \mathrm{~mm} /$ day or less. ${ }^{22}$ Knowing the expected rate of growth of a normal gestational sac allows a followup ultrasound to be scheduled when a specific discriminatory size should be reached. For example if the MSD is $6 \mathrm{~mm}$, then a repeat ultrasound to detect cardiac activity should be performed approximately 10 days later. At this time the MSD is expected to have grown to $16 \mathrm{~mm}$, which is the size at which normal cardiac activity should be detected.

Doppler can be used to distinguish a gestational sac from an intrauterine fluid collection, such as a pseudogestational associated with an ectopic pregnancy. Flow around a true gestational sac, whether normal or abnormal, is usually high velocity with a low-resistance pattern. ${ }^{4}$ In contrast, the flow around a pseudogestational sac is either absent or low velocity.

A single quantitative hCG level is not helpful in distinguishing between a viable and nonviable intrauterine pregnancy. However, an hCG level that is higher than expected given the size of an intrauterine sac, suggests an ectopic pregnancy. Serial hCG levels are often useful for distinguishing a viable pregnancy from a nonviable pregnancy; especially since a falling hCG level predicts pregnancy failure with certainty.

Serum progesterone levels may prove to be useful for assessing pregnancy viability. Studies evaluating the value of progesterone in early pregnancy have shown that a level greater than $25 \mathrm{nmol} / \mathrm{L}$ is associated with a viable pregnancy. ${ }^{23}$ These results need to be confirmed before a low progesterone level can be used to definitively diagnosis a nonviable pregnancy in women with an empty gestational sac. $^{1}$

\section{Detecting an Embryo}

As previously mentioned, an embryo with cardiac activity should be seen when the MSD exceeds $25 \mathrm{~mm}$ using a transabdominal probe, and $16 \mathrm{~mm}$ with a higher frequency vaginal probe. ${ }^{11,12}$

Usually cardiac activity is detected by the time an embryo is seen. However, in some cases cardiac activity is not detected because of the small size of an embryo. Using transabdominal ultrasound cardiac activity should be seen when the embryonic length exceeds $9 \mathrm{~mm}$. With transvaginal ultrasound, cardiac activity should be seen once the embryonic length exceeds 4 to $5 \mathrm{~mm} .{ }^{24}$ Similar to the growth of a gestational sac, a normal embryo is expected to increase in size by $1 \mathrm{~mm} /$ day. ${ }^{14}$ For this reason a follow-up ultrasound to detect cardiac activity should be considered when the embryonic length is expected to exceed the discrimatory length. When an embryo exceeds the discriminatory length of 4 to $5 \mathrm{~mm}$ and cardiac activity is absent by transvaginal ultrasound, an embryonic demise is highly likely. Obviously, this diagnosis should only be made with caution and absolute certainty. In these cases the absence of cardiac activity should be confirmed with M mode and a second examiner before the diagnosis of an embryonic demise is made. Again if there is any question about the diagnosis, a follow-up ultrasound should be obtained.

The presence of cardiac activity is a favorable prognostic finding. The risk of pregnancy loss when cardiac activity is found in an asymptomatic woman greater than 8 weeks gestation is only $2 \% .^{24}$

In 1995 the Board of the Faculty of Clinical Radiology of the Royal College of Radiologists and the Council of the Royal College of Obstetricians and Gynecologists mandated that when the death of an embryo is suspected, two transvaginal scans separated by a minimum of 7 days should be performed to confirm the diagnosis of an early pregnancy failure. ${ }^{25} \mathrm{~A}$ two-week interval is required when the MSD is 
less than $15 \mathrm{~mm}$ or the CRL is less than $10 \mathrm{~mm}$, before a pregnancy failure can be diagnosed. This policy would likely decrease the risk of an incorrect diagnosis, but might lead to more ultrasound exams and a prolonged period of anxiety during which a woman wonders, if her pregnancy is viable. Attempts have been made to identify factors that are diagnostic of an early pregnancy failure in order to minimize the number of ultrasound exams, the time to diagnosis, and the associated anxiety in the event of a threatened pregnancy failure.

\section{ULTRASOUND FINDINGS ASSOCIATED WITH AN INCREASED RISK OF EARLY PREGNANCY} FAILURE

Approximately $15 \%$ of all clinically recognized pregnancies are lost. ${ }^{4}$ When clinically unrecognized pregnancies are included, the rate of early pregnancy loss increases to $31 \%{ }^{26}$ The risk of pregnancy loss decreases with advancing gestational age. For asymptomatic women less than 6.5 weeks gestation, the incidence of pregnancy loss is between 7 to $24 \%$. After 8 weeks gestation the loss rate in asymptomatic women decreases to 2 to $3 \%{ }^{4}$

Vaginal bleeding is associated with an increased risk of pregnancy loss. Vaginal bleeding complicates approximately $25 \%$ of pregnancies. ${ }^{4}$ In early pregnancy, bleeding is often secondary to implantation of the blastocyst into endometrium. If bleeding occurs after the time of implantation, placental separation is the most likely cause. In general, the incidence of pregnancy loss after firsttrimester bleeding and before sonographic evaluation for fetal viability is about $50 \% .{ }^{27}$ Not surprisingly, the risk of pregnancy loss is directly proportional to the reported severity of bleeding. A smaller than expected gestational sac in a woman with vaginal bleeding has been associated with an increased risk of miscarriage. ${ }^{28}$ If a viable fetus is seen at an ultrasound performed after first trimester bleeding, the patient can be reassured that $98 \%$ of such pregnancies will continue beyond 24 weeks gestation. ${ }^{29}$ However, first trimester bleeding is an independent risk factor for adverse pregnancy outcomes, such as preeclampsia, preterm delivery, placental abruption, intrauterine growth restriction, cesarean delivery, and premature rupture of membranes, and the risk is directly proportional to the amount of bleeding.

Several ultrasound findings have been identified that increase the likelihood of an early pregnancy failure. These include an intrauterine blood collection, a slow heart rate, a small gestational sac size, a smaller than expected embryo, an abnormal appearing yolk sac, an abnormal amnion and amniotic cavity, and several maternal factors. Although, none of these findings is diagnostic of a failed pregnancy, they have been associated with an increased risk of pregnancy failure. Therefore these findings might useful in counseling patients, and in deciding the time and type of follow-up.

\section{Intrauterine Blood}

A collection of blood within the uterus is not an uncommon finding, especially in patients with first trimester bleeding (Figure 5). The finding of intrauterine blood is becoming more common with the improved resolution of ultrasound equipment and the increasing indications for first trimester ultrasound exams. The incidence of intrauterine hematomas in the general population is $3.1 \%{ }^{30}$ In high-risk patient populations, such as those with a history of recurrent miscarriage or threatened abortion, the incidence ranges from 4 to $22 \%$. The ability to detect intrauterine blood depends on the size and age of the clot. A recently formed blood clot is often difficult to identify because of its similar echogenicity to placental tissue. With time, clot formation results in sonolucent areas which are more easily recognized as distinct from the surrounding tissue. Approximately $70 \%$ of subchorionic hematomas resolve by the end of the second trimester. ${ }^{30}$ The risk of pregnancy loss with first trimester bleeding and an intrauterine hematoma is controversial; while some studies have found an increased risk of pregnancy loss, others have not. ${ }^{4}$ In some studies the size of the clot has not been shown to correlate with the outcome of the pregnancy. ${ }^{30}$ However, other studies have demonstrated an increased risk of loss with a larger intrauterine clot, early gestational age, and advanced maternal age. The presence of cardiac activity in pregnancies complicated by vaginal bleeding is reassuring. For pregnancies that continue beyond the first trimester, there is evidence to suggest that an intrauterine hematoma is associated with an increased risk

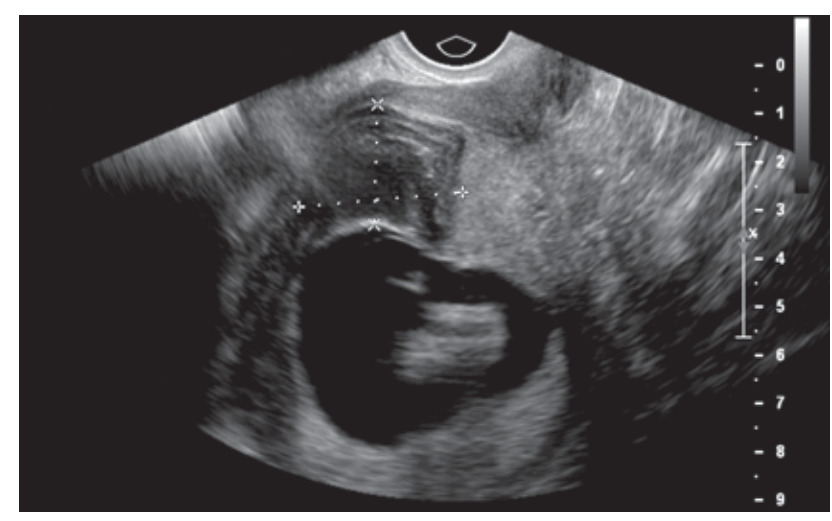

Figure 5: Intrauterine hematoma in the first trimester 
of preterm delivery ranging from 12 to $32 \% .{ }^{30}$ Other complications include intrauterine growth restriction, preeclampsia, placental abruption, manual removal of the placenta, and delivery by cesarean section for fetal distress.

\section{Heart Rate}

High frequency transvaginal ultrasound can detect cardiac activity as early as 34 days of gestation. ${ }^{13}$ During the first trimester, cardiac rates vary with gestational age. Prior to 6 weeks, the heart rate is relatively slow and averages between 100 and 115 beats per minute (BPM). ${ }^{4}$ However, heart rates of 82 BPM at 5 weeks gestation and 96 BPM at 6 weeks gestation have been reported with normal outcomes. ${ }^{31}$ Between 34 to 56 days of gestation, the embryonic heart rate increases by approximately 4 beats/min/day. ${ }^{32}$ The embryonic heart-rate peaks at 8 weeks gestation between 144 and 159 BPM, and after 9 weeks the rate plateaus at 137 to 144 BPM. ${ }^{4}$ Unlike the fetal heart-rate, there is minimal variation in the embryonic heart rate, so that a reliable heart rate can be obtained from a single measurement.

It is known that a slow heart rate is a risk factor for miscarriage. Specifically, a heart rate less than 100 BPM before 6.2 weeks, and less than 120 BPM between 6.3 and 7.0 weeks, has been associated with an increased rate of pregnancy loss. ${ }^{33}$ Embryonic heart-rates less than 80 BPM before 6.3 weeks gestation, and below 100 BPM between 6.3 to 7 weeks gestation have loss rates approaching $100 \%$. An embryo with a slow heart-rate that survives the first trimester has a relatively good prognosis. However, these pregnancies may be at increased risk for congenital anomalies and aneuploidy. An abnormally high heart-rate has not been shown to be a risk factor for miscarriage.

\section{Small Gestational Sac}

A small gestational sac is a rare finding during the first trimester. A small sac has been defined as a difference between the MSD and the CRL of less than $5 \mathrm{~mm} .{ }^{34}$ First trimester oligohydramnios should be distinguished from oligohydramnios that occurs later in pregnancy because at this early gestational age it is chorionic fluid rather than amniotic fluid that is measured. When a small sac is diagnosed, the incidence of spontaneous loss ranges is up to $94 \%$ even in the presence of normal cardiac activity. There is no evidence to suggest that a large gestational sac, suggestive of first trimester polyhydramnios, is predictive of a poor outcome.

\section{Abnormal Yolk Sac}

A normal yolk sac has a maximum diameter of $6 \mathrm{~mm}$ at 10 weeks gestation. While some studies have found that an abnormal yolk sac diameter is associated with subsequent pregnancy failure, other studies have not found this association. ${ }^{4}$

An abnormal appearance of the yolk sac, such as an irregular shape, calcifications, increased echogenicity, or a duplicated yolk sac are all associated with early pregnancy failure. ${ }^{35,36}$

\section{Abnormal Amnion}

In a normal pregnancy the amnion is usually seen after the embryo is identified. As the embryo grows, the mean amnionitic sac diameter and the embryonic length are similar. An amnion that is easily visualized because of increased thickness or echogenicity is often associated with early pregnancy failure. In addition, visualization of the amnion without an embryo or an amnion with a diameter that exceeds the CRL by much more than $1 \mathrm{~mm}$ is also associated with an increased risk of pregnancy failure. ${ }^{21}$

\section{Maternal Factors}

Maternal factors associated with an increased risk of pregnancy loss include advanced maternal age, a history of recurrent pregnancy loss, and structural abnormalities of the uterus. Most of these factors can be assessed by a thorough history and exam. However, structural abnormalities of the uterus often require diagnostic imaging.

Structural abnormalities of the uterus can be either congenital or acquired. Congenital malformations, such as a septate uterus, have a strong association with early pregnancy loss. It is thought that a poorly vascularized septum is unable support an early pregnancy. Acquired malformations, such as synechiae and fibroids, are also associated with an increased risk of early pregnancy loss.

\section{Doppler Studies}

Experimental studies have evaluated the role of first trimester Doppler in distinguishing between a normal $v s$ an abnormal pregnancy. Results of studies using Doppler to evaluate uteroplacental blood flow have been conflicting in terms of predicting pregnancy outcome. Doppler evaluation of corpus luteum blood flow during the first trimester has not been shown to predict pregnancy outcome. Similarly, Doppler 
studies of areas surrounding an intrauterine sac have not been able to reliably distinguish a gestational sac from the pseudogestational sac of an ectopic pregnancy. Because studies evaluating the usefulness of Doppler in the first trimester have been conflicting, and because of concern about the increased energy level of Doppler ultrasound during this period of development, the routine use of Doppler in the first trimester should be avoided. ${ }^{4}$

\section{FIRST TRIMESTER ULTRASOUND IN MULTIFETAL GESTATIONS}

Since 1980 twin births have increased by $65 \% .{ }^{37}$ Currently, it is estimated that $3 \%$ of all births in the US are twins. The increase in multiple gestations has been attributed to an increase in infertility treatments and an aging maternal population, both of which are associated with a higher risk of multifetal gestations.

Twin gestations are either dizygotic or monozygotic. A dizygotic twin pair results from fertilization of two separate ova, while a monozygotic twin pair results from fertilization of a single ovum. Approximately two-thirds of spontaneously conceived twin pregnancies are dizygotic and onethird are monzygotic. Dizygotic twins have their own placentas and amniotic sacs, and are therefore dichorionicdiamniotic. The number of placentas and amniotic sacs in a monozygotic twin pair depends on when division of the zygote occurs relative to formation of the chorion and amnion. The chorion is formed 4 days after fertilization and then the amnion is formed at 8 days after fertilization. Approximately 25\% of monozygotic twins divide within 4 days of fertilization, prior to differentiation of the amnion and chorion, and are thus dichorionic-diamniotic. Seventyfive percent of the time the zygote divides between day 4 , after the chorion is formed, and day 8, prior to formation of the amnion. This results in a monochorionic-diamniotic twin pair, in which there is a single placenta and two separate amniotic sacs. When division occurs more than 8 days after fertilization, the chorion and amnion have already differentiated, so that a monochorionic-monoamniotic twin pair is formed. Approximately $1 \%$ of monozygotic twins are monochorionic-monoamniotic. Rarely, the zygote divides more than 13 days after fertilization. After 13 days, division is usually incomplete and results in conjoined twins. It is well known that the risks of perinatal morbidity and mortality are increased in a multiple gestation and that the risk increases with each additional fetus. Furthermore, the perinatal mortality is two to three times higher in monochorionic twins than in dichorionic twin pregnancies. For this reason, knowledge of chorionicity and amnionicity is essential in managing multiple gestations. ${ }^{38}$

\section{First Trimester Determination of Chorionicity and Amnionicity}

Ultrasound assessment of chorionicity and amnionicity is most accurate in the first trimester, and may not be possible later in gestation. In the first trimester, chorionicity can be determined by counting the number of gestational sacs. Amnionicity can be assigned based on the number of amnions or yolk sacs. ${ }^{38}$

\section{Chorionicity}

Counting the number of gestational sacs is an accurate method for determining chorionicity in the first trimester. This is true because the gestational sac is actually the chorionic cavity filled with chorionic fluid. Therefore the number of chorionic cavities reflects the chorionicity of the pregnancy. The optimal time for determining chorionicity using transvaginal ultrasound is between 6 to 10 weeks gestation. Although, the gestational sac can usually seen by transvaginal ultrasound at 5 weeks, evaluation of multifetal gestations prior to 6 weeks can result in an undercounting of gestational sacs in $15 \%$ of pregnancies. ${ }^{39}$ After 10 weeks gestation, the amniotic membrane fills the chorionic cavity. In a diamniotic twin pair the amniotic membranes become opposed to form an intertwin membrane (Figure 6). After this time, it is no longer possible to determine chorionicity by simply counting the number of gestational sacs. However, the thickness of the intertwin membrane in the first trimester may be useful for diagnosing chorionicity. The intertwin membrane in a dichorionic pregnancy is composed of two layers of amnion and two layers of chorion, while a monochorionic intertwin membrane is composed of two layers of amnion without a chorion. An intertwin membrane that appears hyperechoic and measures more than $1.5 \mathrm{~mm}$ is consistent with a dichorionic twin pregnancy, while a thin membrane measuring less than 1.5 $\mathrm{mm}$ is more likely to be seen in a monochorionic gestation. ${ }^{40}$ Other methods for determining chorionicity in the first trimester include counting the number of placentas and assessing whether placental tissue extends into the base of the intertwin membrane. The finding of placental tissue extending into the base of the membranes, known as the lambda sign or placental peak sign, is suggestive of a dichorionic pregnancy (Figure 7). In monochorionic pregnancies there is no extension of placental tissue within 


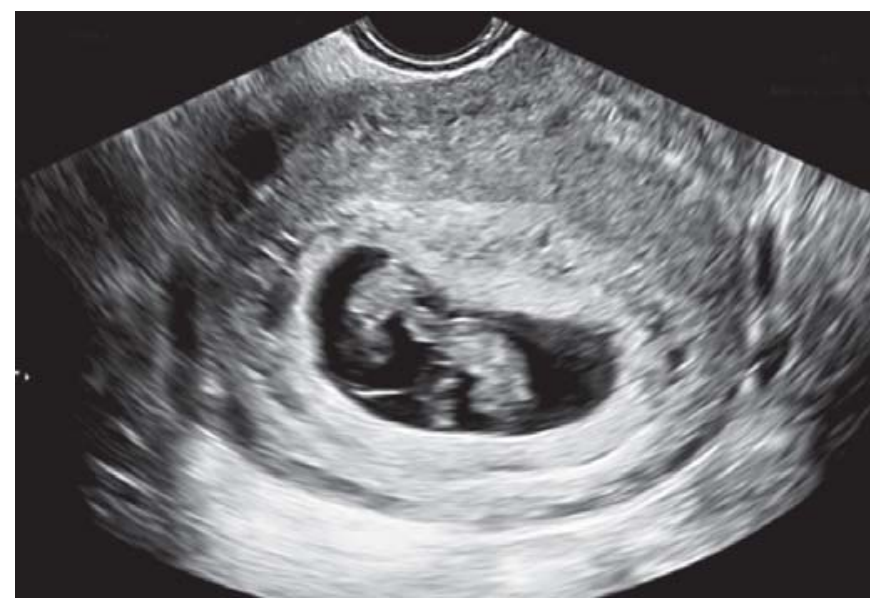

Figure 6: Monochorionic twin membranes opposed

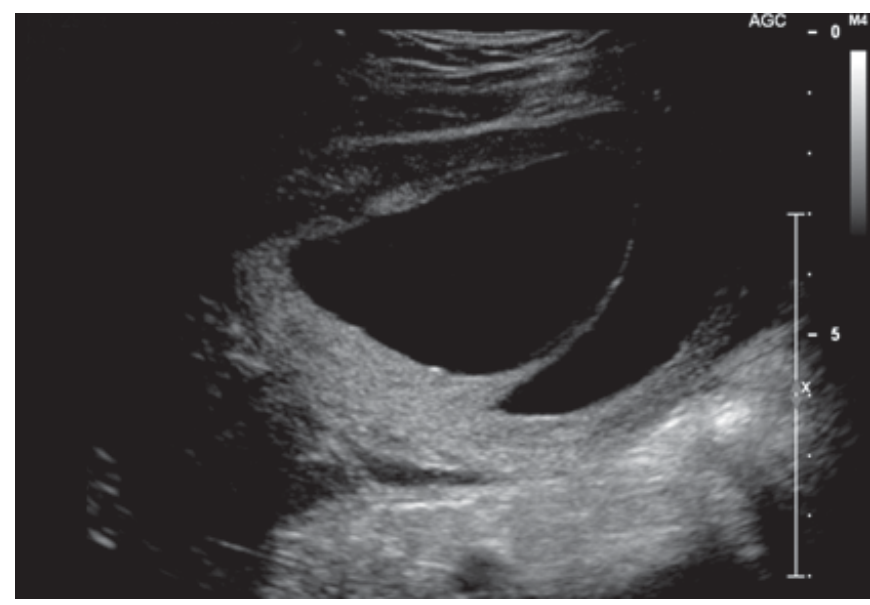

Figure 7: Lambda or placental peak sign of a dichorionic twin gestation

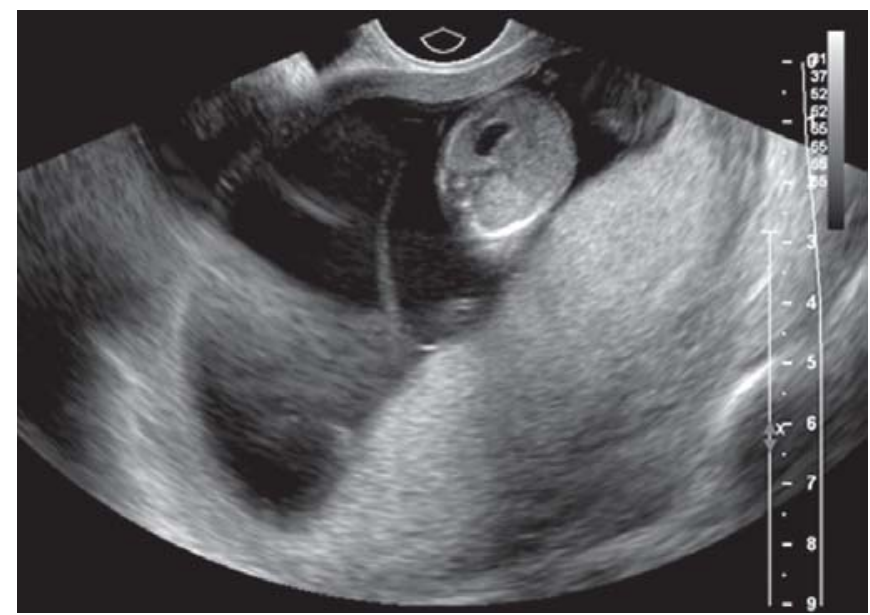

Figure 8: T sign of a monochorionic twin gestation

the membranes, which results in the appearance of a "T" where the membranes attach to the placenta (Figure 8). Pathology studies have shown that the ultrasound findings of a lambda sign or two separate placentas has a sensitivity of $97.4 \%$ and a specificity of $100 \%$ in the prediction of dichorionicity, while the $\mathrm{T}$ sign has a sensitivity of $100 \%$ and a specificity of $98.2 \%$ in predicting monochorionicity. ${ }^{40}$

\section{Amnionicity}

As a rule, all dichorionic pregnancies are diamniotic. Monochorionic twins can be either diamniotic or monoamniotic depending on if the zygote divides before or after the amnion differentiates. At 6 weeks gestation, amnionicity can be determined by counting the number of embryos within each gestational sac. If only one embryo is seen in each gestational sac, then the amniocity equals the chorionicity. However, if two embryos are seen within a single gestational sac, then the pregnancy is either monochorionic-diamniotic or monochorionic-monoamniotic. In this case the amniocity can be determined by counting the number of yolk sacs or amnions within the gestational sac. Counting the number of yolk sacs is an accurate method for determining amnionicity before the amnion can be visualized. Monoamniotic twins have a single yolk sac, whereas diamniotic twins usually have two (Figure 9). Because the yolk sac divides shortly before the amnion, up to $15 \%$ of monochorionic diamniotic twins may have a single yolk sac. ${ }^{41}$ The yolk sac is first visible by transvaginal ultrasound between 5 to 6 weeks gestation. When a single yolk sac is seen, a follow-up ultrasound should be performed to reevaluate the number of yolk sacs or to count the number amnions to exclude a monoamniotic twin gestation. The amniotic membrane is first visualized after 8 weeks gestation by transvaginal ultrasound. If two amnions are seen, the pregnancy is diamniotic. If only one amniotic sac is seen containing two embryos, then the pregnancy is monoamniotic. Monoamniotic twins are associated with high perinatal mortality rates. This increased mortality is secondary to the high

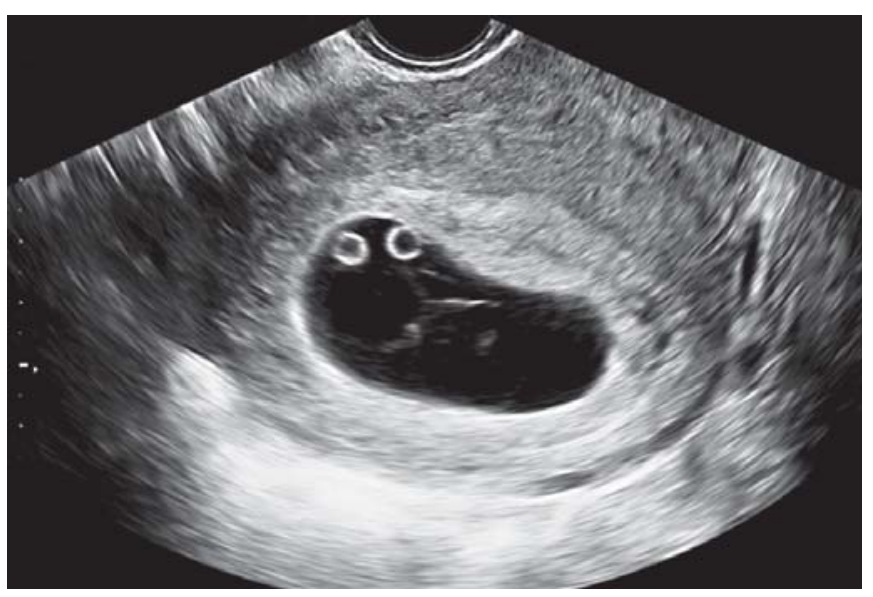

Figure 9: Monochorionic diamniotic twins with two yolk sacs 
prevalence of discordancy for fetal structural anomalies and cord entanglement. Cord entanglement is another way in which monoamniotic twins can be diagnosed in the first trimester.

\section{VANISHING TWIN}

The incidence of early pregnancy loss in multiple gestations has only recently become clear with the advances in early ultrasound imaging. Mathematical estimates have shown that between 10 and $15 \%$ of all live births start as twins. ${ }^{42}$ A meta-analysis of early pregnancy loss in multiple gestations showed that when two gestational sacs are seen, the loss of one twin occurs in $40.5 \%$ of spontaneous pregnancies and in $27.1 \%$ of pregnancies achieved by assisted reproduction. When two embryos are seen, the loss of one twin occurs in $7.3 \%$ of spontaneous pregnancies and in $38 \%$ of pregnancies achieved by assisted reproduction. Overall, $61 \%$ of pregnancies where two gestational sacs or embryos were seen continued as twin gestations. Approximately $40 \%$ of triplet pregnancies with three gestational sacs or embryos will continue beyond the first trimester as a triplet gestation. Vaginal bleeding or spotting is the only complication that has been associated with the loss of an embryo or gestational sac in a multiple gestation. In general, the prognosis of a multiple gestation that has undergone an early spontaneous reduction is favorable. However, the data regarding the prognosis when this complication occurs in monochorionic gestation is limited. There is one report of multicystic encephalomalacia developing in the surviving cotwin of a monochorionic twin pregnancy after a fetal loss at 12 weeks gestation. ${ }^{43}$ When a spontaneous reduction occurs in a monochorionic gestation prior to twelve weeks, it is not known if the cotwin is at increased risk of loss or morbidity.

\section{CONCLUSION}

It is likely that the technological development of ultrasound will continue and that increases in ultrasound frequency will further improve image resolution of early pregnancies. Additional technology, such as three- and four-dimensional ultrasonography, will also likely improve our ability to assess early pregnancy viability and multifetal gestations.

\section{REFERENCES}

1. American College of Obstetricians and Gynecologists. Ultrasonography in pregnancy. ACOG Practice Bulletin No. 58. Obstet Gynecol 2004;104:1449-58.
2. Ferrazzi E, Garbo P, Sulpizio L, Setti L, Buscaglia M. Miscarriage diagnosis and gestational age estimation in the early first trimester of pregnancy: Transabdominal versus transvaginal sonography. Ultrasound Obstet Gynecol 1993;3:36-41.

3. Filly RA, Hadlock FP. Sonographic Determination of Menstrual Age. In Callen, PW (Ed): Ultrasonography in Obstetrics and Gynecology (4th ed), Philadelphia: WB Saunders, 2000:146-70.

4. Laing FC, Frates MC. Ultrasound Evaluation During the First Trimester of Pregnancy. In Callen, PW (Ed): Ultrasonography in Obstetrics and Gynecology (4th ed), Philadelphia: WB Saunders 2000;105-45.

5. Yeh HC, Goodman JD, CarrL, Rabinowitz JG. Intradecidual sign: A US criterion of early intrauterine pregnancy. Radiology 1986;161:463-67.

6. Laing FC, Brown DL, Price JF, Teeger S, Wong ML. Intradecidual sign: Is it effective in diagnosis of an early intrauterine pregnancy? Radiology 1997;204:655-60.

7. Bradley WG, Fiske CE, Filly RA. The double sac sign of early intrauterine pregnancy: Use in exclusion of ectopic pregnancy. Radiology 1982;143:223-26.

8. Lazarus E. What's new in first trimester ultrasound. Radiol Clin N Am 2003;41:663-79.

9. Jauniaux E, Jurkovic D, Henriet Y, Rodesch F, Hustin J. Development of the secondary human yolk sac: Correlation of sonographic and anatomical features. Hum Reprod 1991;6: 1160-66.

10. Nyberg DA, Mack LA, Harvey D, Wang K. Value of the yolk sac in evaluation of early pregnancies. J Ultrasound Med 1988; 7:129-35.

11. Levi CS, Lyons EA, Lindsay DJ. Early diagnosis of nonviable pregnancy with transvaginal US. Radiology 1988;167:383-85.

12. Nyberg DA, Laing FC, Filly RA. Threatened abortion: Sonographic distinction of normal and abnormal gestation sacs. Radiology 1986;158:397-400.

13. Coulam CB, Britten S, Soenksen DM. Early (34-56 days from last menstrual period) ultrasonographic measurements in normal pregnancies. Hum Reprod 1996;11:1771-74.

14. Lasser DM, Peisner DB, Vollebergh J, Timor-Tritsch I. Firsttrimester fetal biometry using transvaginal sonography. Ultrasound Obstet Gynecol 1993;3:104-08.

15. Blaas HG, Eik-New SH, Kiserud T, Hellevik LR. Early development of the abdominal wall, stomach and heart from 7 to 12 weeks of gestation: A longitudinal ultrasound study. Ultrasound Obstet Gynecol 1989;161:831-33.

16. Zimmer EZ, Chao CR, Santos R. Amniotic sac, fetal heart area, fetal curvature, and other morphometrics using first trimester vaginal ultrasonography and color Doppler imaging. J Ultrasound Med 1994;13:685-90.

17. Bennet KA, Crane JM, O’Shea P, Lacelle J, Hutchens D, Copel JA. First trimester ultrasound screening is effective in reducing postterm labor induction rates: A randomized controlled trial. Am J Obstet Gynecol 2004;190:1077-81.

18. Benson CB, Doubilet PM. Fetal measurements—normal and abnormal fetal growth. In Rumack CM, Wilson SR, Charboneau JW (Eds): Diagnostic Ultrasound (2nd ed). St. Louis: CV Mosby, 1998;1013-31.

19. Robinson HP, Fleming JE. A critical evaluation of sonar CRL measurements. BJOG 1975;82(9):702-10. 
20. Goldstein SR, Wolfson R. Transvaginal ultrasonographic measurement of early embryonic size as a means of assessing gestational age. J Ultrasound Med 1994;13:27-31.

21. Nyberg DA, Filly RA. Predicting pregnancy failure in "empty" gestational sacs. Ultrasound Obstet Gynecol 2003;21:9-12.

22. Nyberg DA, Mack LA, Laing FC, Patten RM. Distinguishing normal from abnormal gestational sac growth in early pregnancy. J Ultrasound Med 1987;6:23-27.

23. Elson J, Salim R, Tailor A, Banerjee S, Zosmer N, Jurkovic D. Prediction of early pregnancy viability in the absence of an ultrasonically detectable embryo. Ultrasound Obstet Gynecol 2003;21:57-61.

24. Cashner KA, Christopher CR, Dysert GA. Spontaneous fetal loss after demonstration of a live fetus in the first trimester. Obstet Gynecol 1987;70:827-30.

25. Hately W, Case J, Campbell S. Establishing the death of an embryo by ultrasound: Report of a public inquiry with recommendations. Ultrasound Obstet Gynecol 1995;5:353-57.

26. Wilcox AJ, Weinberg CR, O'Connor JF, Baird DD, Schlatterer JP, Canfield RE, Armstrong EG, Nisula BC. Incidence of early loss of pregnancy. N Engl J Med 1988;319(4):189-94.

27. Chung TKH, Sahota DS, Lau TK, Mongelli JM, Spencer JAD, Haines CJ. Threatened abortion: Prediction of viability based on signs and symptoms. Aust N Z J Obstet Gynaecol 1999;39: 443-47.

28. Falco P, Zagonari S, Gabrielli S, Bevini M, Pilu G, Bovicelli L. Sonography of pregnancies with first-trimester bleeding and a small intrauterine gestational sac without a demonstrable embry. Ultrasound Obstet Gynecol 2003;21:62-65.

29. Weiss JL, Malone FD, Vidaver J, Ball RH, Nyberg DA, Comstock CH, Hankins GD, Berkowitz RL, Gross SJ, Dugoff L, Timor-Tritsch I, D’Alton ME. Threatened abortion: A risk factor for poor pregnancy outcome, a population-based screening study. Am J Obstet Gynecol 2004;190:745-50.

30. Nagy S, Bush M, Stone J, Lapinski R, Gardo S. Clinical significance of subchorionic and retroplacental hematomas detected in the first trimester of pregnancy. Obstet Gynecol 2003;102:94-100.
31. Merchiers EH, Dhont M, De Sutter PA, Beghin CJ, Vandekerckhove DA. Predictive value of early embryonic cardiac activity for pregnancy outcome. Am J Obstet Gynecol 1991;165:11-14.

32. Britten S, Soenksen DM, Bustillo M, Coulam CB. Very early (24-56 days from last menstrual period) embryonic heart-rate in normal pregnancies. Hum Reprod 1994;9:2424-26.

33. Doubilet PM, Benson CB. Embryonic heart-rate in the early first trimester: What rate is normal? J Ultrasound Med 1995;14:431-34.

34. Bromley B, Harlow BL, Laboda LA, Benacerraf BR. Small sac size in the first trimester: A predictor of poor fetal outcome. Radiology 1991;178:375-77.

35. Harris RD, Vincent LM, Askin FB. Yolk sac calcification: A sonographic finding associated with intrauterine embryonic demise in the first trimester. Radiology 1988;166:109-10.

36. Barzilai M, Lyons EA, Levi CS, Lindsay DJ. Vitelline duct cyst or double yolk sac. J Ultrasound Med 1989;8:523-26.

37. Martin JA, Hamilton BE, Sutton PD, Ventura SJ, Menacker F, Munson ML. Births: Final data for 2002. Natl Vital Stat Rep 2003;52:1-113.

38. Hall JG. Twinning. Lancet 2003;362:735-43.

39. Doubilet PM, Benson CB. "Appearing twin”: Undercounting of multiple gestations on early first trimester sonogram. J Ultrasound Med 1998;17:199-203.

40. Carroll SG, Soothill PW, Abdel-Fattah SA, Porter H, Montague I, Kyle PM. Prediction of chorionicity in twin pregnancies at 10-14 weeks of gestation. BJOG 2002;109:182-86.

41. Shen O, Samueloff A, Beller U, Rabinowitz R. Number of yolk sacs does not predict amnionicity in early first-trimester monochorionic multiple gestations. Ultrasound Obstet Gynecol 2006;27:53-55.

42. Landy HJ, Keith LG. The vanishing twin: A review. Hum Reprod Update 1998;4:177-83.

43. Weiss JL, Cleary-Goldman J, Tanji K, Budorick N, D’Alton ME. Multicystic encephalomalacia after first-trimester intrauterine fetal death in monochorionic twins. Am J Obstet Gynecol 2004;190:563-65. 